\title{
Habiletes Sociales Acquises Au Sein Des Familles Et Resilience Au Passage A L'acte Delictueux Chez Des Jeunes Vivant Dans Des Quartiers Defavorises A Abobo. (Abidjan)
}

\author{
Dr. Traore Massandjé \\ Dr. Crizoa Hermann \\ Maître Assistant à l'UFR Criminologie \\ N'goran N'faissoh Franck Stéphane \\ Doctorant à l'UFR Criminologie, \\ Université Félix Houphouët-Boigny Cocody, Côte d'Ivoire
}

Doi:10.19044/esj.2018.v14n29p315 URL:http://dx.doi.org/10.19044/esj.2018.v14n29p315

\begin{abstract}
This study aims to explain the link between the social skills acquired within families and the resilience to the criminal act in young people living in disadvantaged neighborhoods in Abobo. The research was carried out in Abobo commune and involved 74 participants from different social categories. The collection of information relating to the object of study was based on questionnaire, interview and observation. The information collected was analyzed from a quantitative and qualitative point of view. The results of the study indicate that youth who are resilient to delinquency in the community are of all ages and both sexes. The study shows that the resilience to the criminal act in certain young people living in the precarious neighborhoods of the Abobo commune is explained by the ability to ask for help, self-control, development of a sense of autonomy and a projection into the future.
\end{abstract}

Keywords: Social skills, family, resilience, crime, youth, precarious neighborhoods.

\section{Resume}

Cette étude vise à expliquer le lien entre les habiletés sociales acquises au sein des familles et la résilience au passage à l'acte délictueux chez des jeunes vivant dans des quartiers défavorisés à Abobo. Les recherches ont été effectuées dans la commune d'Abobo et ont porté sur 74 participants issus de différentes catégories sociales. La collecte d'informations relatives à l'objet d'étude s'est faite à partir de questionnaire, d'entretien et de l'observation. Les informations recueillies ont été analysées d'un point de vue quantitatif et 
qualitatif. Les résultats de l'étude indiquent que les jeunes qui font preuve de résilience à la délinquance dans la commune sont de tous les âges et des deux sexes. L'étude montre que la résilience au passage à l'acte délictueux chez certains jeunes vivant dans les quartiers précaires de la commune d'Abobo s'explique par la capacité à demander de l'aide, la maîtrise de soi, le développement d'un sentiment d'autonomie et d'une projection dans l'avenir.

Mots-Cles : Habiletés sociales, famille, résilience, passage à l'acte délictueux, jeunes, quartiers précaires.

\section{I/ INTRODUCTION}

La commune d'Abobo compte plus d'une dizaine de quartiers précaires et un (1) bidonville. Les quartiers ne sont, pour la plupart, pas viabilisés. Avec une population croissante et une demande de logement de plus en plus forte, environ $60 \%$ de la population de ladite commune vivent dans ces quartiers précaires. De plus, les braquages y représentent $45 \%$ des crimes commis (Sommer, Guébo, Vignol, Maréchal, Sublet \& Kuria, 2012). Les violences et voies de faits de même que les vols toutes catégories, représentent 95.36\% de l'ensemble des infractions commises dans ladite commune et les individus qui commettent les actes criminels dans cette commune sont pour la plupart des jeunes, en majorité, de sexe masculin dont l'âge est compris entre 10 et 20 ans (N'goran, 2014).

Selon les statistiques criminelles de la Préfecture de Police d'Abidjan (2016), le phénomène de la criminalité dans le District d'Abidjan n'a cessé de croître et de se diversifier de 2014 à 2015. Les statistiques recueillies auprès de la Police font respectivement état de 628 et de 1138 affaires criminelles en 2014 et en 2015, avec une prépondérance aux actes de vols avec effraction ${ }^{1}$. Abobo constitue à cet effet une "zone à risque". Cependant, en dépit de ce constat, il est à notifier que dans cette commune, même s'il est avéré que certains jeunes passent à l'acte délictueux, il est tout de même nécessaire de souligner que d'autres ne s'y prêtent pas au regard des statistiques évoquées. Ceci indique qu'il existe des facteurs qui pourraient expliquer la résilience au passage à l'acte délictueux chez des jeunes vivant à Abobo.

Anaut (2005), affirme que la résilience n'est pas acquise une fois pour toutes, mais en constant développement et l'on observe l'émergence du processus résilient à tout âge, y compris dans la vieillesse. Glaeser, Sacersote et Scheinkman (1996), quant à eux, estiment que la décision d'un individu de commettre ou non une infraction dépend du choix délinquant réalisé par son entourage. Car, en dépit de la précarité des conditions de vie de certains jeunes, il en existe d'autres qui parviennent à les surmonter. Des recherches ont

\footnotetext{
${ }^{1}$ Source : Service statistique de la Préfecture de Police d'Abidjan
} 
montré qu'un certain nombre d'enfants de milieux socio-économiquement faibles, bien que confrontés à des conditions environnementales à première vue très défavorables sur le plan socio-économique, étaient bien adaptés et performant sur le plan scolaire (Kouakou Oi Kouakou, 2016), voire même dans la vie active (Terrise \& Larose, 2001). Le processus de résilience peut s'enclencher plus facilement au fur et à mesure que les personnes vivent différentes situations problématiques et viennent à bout des adversités rencontrées (Ionescu, 2012). Pour ces auteurs, il s'agit donc d'enfants « atypiques », dans leur milieu, dont le cas infirme le déterminisme sociologique. Les enfants font preuve de résilience selon les ressources auxquelles ils ont accès au sein de leur famille ou de leur collectivité et selon leurs propres ressources émergentes (Masten, 2001). La résilience dépend donc des habiletés familiales et de celles des autres aspects de l'environnement social à compenser les effets des circonstances adverses.

Le concept de résilience est transdisciplinaire et polysémique. D'abord utilisé en mécanique pour désigner la résistance d'un matériau à un choc $^{2}$ (Maret et Cadoul, 2008), ce terme est ensuite récupéré en psychologie comportementaliste et développementaliste et en neuro-psychiatrie (Thomas, 2008). Dans ces domaines, il est convenu que la résilience représente [...] la capacité de l'individu de se construire et de vivre de manière satisfaisante malgré les difficultés et les situations traumatiques auxquelles il peut être confronté au cours de son existence (Tychey, 2001). C'est ainsi que les enfants peuvent réussir malgré le risque, grâce à divers atouts dont plusieurs sont extérieurs à leur personnalité comme le soutien des parents, des grandsparents, ou des communautés qui fonctionnent bien et ont des liens étroits (Luthar, 2013). Ce qui provoque l'attachement et donne confiance à l'enfant, ce n'est pas la satisfaction des besoins, mais plutôt la mise à la disposition de l'enfant d'une base de sécurité (Gonnet, Koffi \& Cyrulnik, 2010). Le processus de résilience est un phénomène complexe qui implique l'interaction de facteurs psychoaffectifs, relationnels et sociaux avec les caractéristiques internes du sujet (processus défensifs, personnalité, etc.) (Anaut, 2005).

Il s'agit donc de savoir dans cette étude, pourquoi dans le même quartier précaire, certains jeunes résistent au passage à l'acte délictueux et d'autres non?

Les habiletés sociales acquises au sein des familles expliquent-elles la résilience chez ces jeunes ?

Cette étude vise à montrer le lien entre les habiletés sociales acquises au sein des familles et la résistance au passage à l'acte délictueux chez certains jeunes vivant dans des quartiers défavorisés à Abobo.

\footnotetext{
${ }^{2}$ La plupart des dictionnaires adopte encore aujourd'hui cette définition
} 
Pour atteindre cet objectif, il sera procédé à la vérification de l'hypothèse suivante :

La résilience au passage à l'acte délictueux chez certains jeunes vivant dans les quartiers précaires s'explique par des habiletés sociales acquises au sein de leurs familles.

\section{II/ METHODOLOGIE}

- Terrain d'étude

Le choix de la commune d'Abobo se justifie par sa forte densité démographique qui est de 19230.7692 habitants $/ \mathrm{km}^{2}$ (Densité= nombre d'habitants (1500 000 en 2011)/ superficie (7800 hectares) : source Mairie d'Abobo), favorisant ainsi l'émergence de plusieurs quartiers précaires. Ceci peut entrainer l'accroissement de la délinquance. En outre, dans l'imaginaire populaire, la commune d'Abobo est perçue comme une commune où désordre et violence sont récurrents.

\section{- Population source et échantillon}

La population source est l'ensemble des jeunes résilients vivant dans les quartiers précaires de la commune d'Abobo. Nous avons utilisé une méthode empirique car nous ne disposions d'aucune base de sondage. Plus précisément, il s'agit d'un échantillon de convenance. Le choix de ce type d'échantillon est dû à des raisons pratiques d'accessibilité et de coût. Mais l'inconvénient majeur d'un tel échantillon est qu'il peut parfois nuire à la possibilité de généralisation des résultats à la population mère, car souvent non représentatif de celle-ci. Cette enquête a été menée non seulement dans les quartiers précaires de la commune d'Abobo, mais aussi au centre d'observation des mineurs (COM), sis au sein de la MACA. Ainsi, des jeunes résilients, des jeunes délinquants d'Abobo du COM et les familles des différentes catégories de jeunes, des responsables d'institutions privées ou publiques (BICE, COM, etc.) militant en faveur de la protection et du bien-être de l'enfant ont été interrogés. L'échantillon est composé de 50 jeunes résilients de différents quartiers précaires de la commune d'Abobo; 10 jeunes délinquants d'Abobo du Centre d'Observation des Mineurs, 05 familles dont les enfants ont des comportements pro sociaux, 05 autres familles ayant des enfants délinquants et 04 responsables du BICE et du COM. Le choix des familles ou parents (frères, sœurs, Père, Mère, tuteur, etc.) de jeunes ayant des comportements résilients ou délictueux, dans la population d'enquête, répond au besoin de fournir des informations visant à mieux appréhender l'histoire de vie des jeunes issus des quartiers défavorisés de la commune d'Abobo. Ces familles ont été retrouvées par l'entremise de quelques jeunes d'entre ceux interrogés, qui ont bien voulu que nous échangions avec leurs parents. Ce fut surtout le cas de jeunes résilients. Quant aux familles de jeunes délinquants, nous les 
avons rencontrées lors de leurs visites à leurs enfants ou protégés au sein du COM. Les thèmes abordés dans ces familles étaient le plus souvent relatifs au style éducatif adopté par celles-ci, à la capacité chez leurs enfants à demander de l'aide lorsqu'ils en ont besoin, aux types de soutiens sociaux dont les enfants bénéficient en famille, aux statuts matrimonial et socioprofessionnel des parents et à la précarité socioéconomique ${ }^{3}$ de ces familles.

La prise en compte des responsables d'institutions privées ou publiques (BICE, COM, etc.) militant en faveur de la protection et du bien-être de l'enfant est nécessaire, dans la mesure où, ceux-ci, à partir d'informations que nous pourrons recueillir auprès d'eux, en rapport avec le comportement et le mode de vie des enfants qu'ils ont à leur charge ou qu'ils côtoient le plus souvent, pourraient nous permettre de comprendre davantage les motifs du passage à l'acte délictueux ou non de certains jeunes.

\section{- Instrument de collecte des données et Méthodes d'analyse}

Comme instrument de recueil de données, nous avons utilisé deux (02) questionnaires d'enquête destinés aux jeunes résilients, suivis d'un entretien avec les jeunes délinquants d'Abobo du COM et les familles de chaque catégorie de jeunes pour la collecte des données et une grille d'observation élaborée sur la base de quelques indicateurs. Il s'agit du profil des jeunes à risque (âge, sexe, niveau d'étude, etc.), de la situation socioprofessionnelle des parents des jeunes à risque et des rapports sociaux avec leur environnement immédiat et les groupes de pairs. Ces outils de collecte d'informations constitués respectivement de questions fermés et de questions ouvertes ont permis non seulement de faire l'analyse quantitative de certaines données et aussi l'analyse qualitative des autres, mais aussi de connaître les raisons qui conduisent certains jeunes vivant dans des quartiers défavorisés à Abobo, à résister ou non au passage à l'acte délictueux.

L'analyse quantitative a permis la mesure ou le regroupement des données issues du questionnaire à l'aide de l'outil informatique. Pour mener à bien cette analyse, l'on s'est appuyé sur le logiciel informatique appelé : SPSS version 20. Ainsi, ces données ont été transformées en statistiques descriptives et en distribution de fréquences de moyennes. Cette méthode a permis d'expliquer à partir de calculs, de tableaux statistiques, de graphiques, la résilience à la délinquance des jeunes vivant dans des quartiers défavorisés à Abobo. Quant à l'analyse qualitative, elle a permis de mieux comprendre le vécu des populations en termes de pratiques, significations, perceptions du phénomène étudié et de cerner les logiques des acteurs. Elle a donc permis d'analyser les

\footnotetext{
${ }^{3}$ Habitats de fortune, famille nombreuse, activité socioprofessionnelle à faible revenu, insuffisance d'éclairage public, cadre de vie insalubre, construction anarchique, etc.
} 
données relatives aux avis, opinions, discours et idées exprimées par les personnes interviewées.

\section{III/ RESULTATS}

1. Caractéristiques psychosociales des jeunes résilients et de leurs familles d'appartenance

\subsection{Caractéristiques psychosociales des jeunes résilients}

\subsubsection{Age et sexe des jeunes résilients}

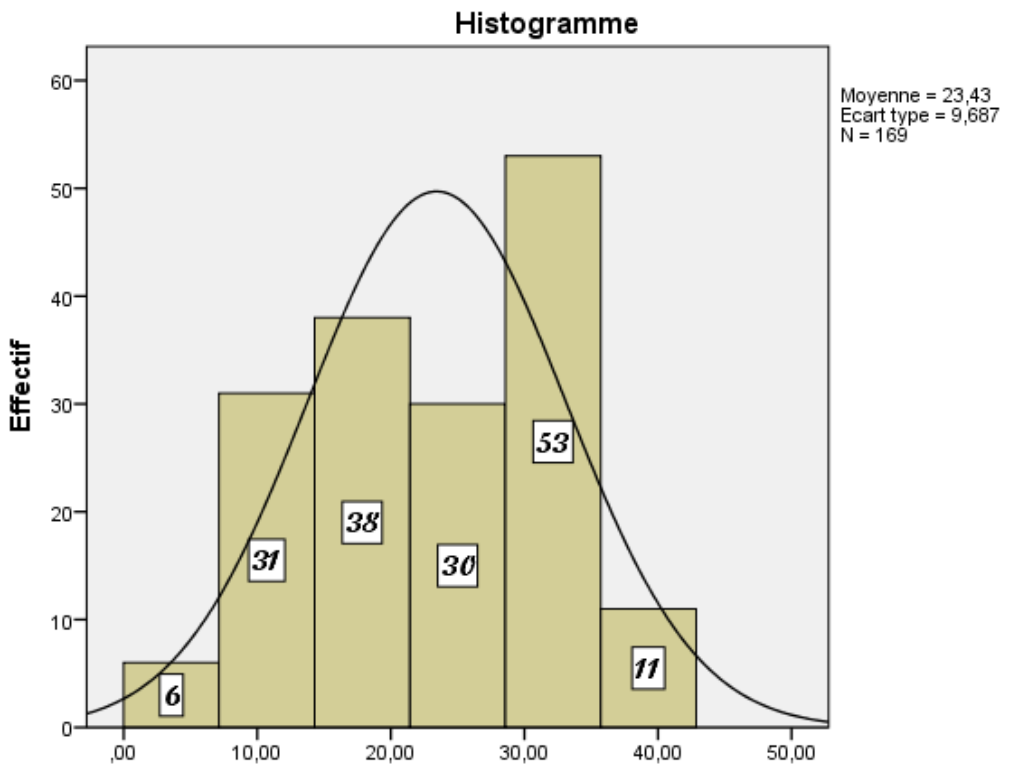

Figure 1: Age des jeunes qui ne commettent pas de crime dans la commune d'Abobo. Source : Enquête, 2016

L'histogramme ci-dessous donne plus de précision sur l'âge des personnes qui ne commettent pas en général des actes de délinquance dans la commune d'Abobo. De même, au regard de la médiane (25) de cette distribution, nous remarquons que $50 \%$ des jeunes qui ne passent pas à l'acte délictueux dans la commune d'Abobo ont au moins 25 ans et $50 \%$ de ces jeunes ont au plus 25 ans.

Aussi à partir de cet histogramme, pouvons-nous affirmer que les jeunes qui font preuve de résilience au passage à l'acte dans la commune d'Abobo appartiennent à toutes les classes d'âge.

Cette courbe est une courbe asymétrique droite avec étalement vers la gauche, car la moyenne, la médiane et le mode n'ont pas relativement la même valeur [Mo (30) > Me $(25)>\bar{X}(23,43)]$. Par conséquent nous pouvons affirmer que la distribution de cette courbe n'est pas normale. Aussi, le Coefficient de variation $\mathrm{CV}=\frac{\text { Ecart type }}{\text { Moyenne }} * 100$, dont la valeur est de $41,26 \%>15 \%$ montre 
que la série est très fortement dispersée autour de sa moyenne. Par ailleurs, ces données statistiques révèlent qu'en général, l'âge des jeunes qui ne commettent pas de crime dans la commune d'Abobo varie entre 30 et 35 ans. A cet âge, selon Sampson et Laub (Koudou, 2006) les activités criminelles sont incompatibles avec un emploi stable, une vie familiale harmonieuse, une éducation adéquate des enfants, etc. En outre Ouimet et Leblanc (Koudou, Id) stipulent que la volonté d'abandonner la carrière criminelle à l'âge adulte provient à la fois d'un processus de maturation et de changements survenus à la situation de vie. Ainsi pour ces auteurs, plus rapide sera l'entrée dans le monde conventionnel par le mariage, le travail et l'abandon de la consommation de drogues plus rapide sera l'abandon de la carrière criminelle.

Pour mieux comprendre l'association entre la résilience et certaines variables ("sexe", "âge", "niveau d'étude" et "Elaboration de projets et projection dans l'avenir") susceptibles d'avoir un impact sur sa survenue, nous avons procédé à la régression logistique de ses variables sur elle. Les données sont contenues dans le tableau ci-dessous :

Tableau 1 : Variables de l'équation

\begin{tabular}{|c|c|c|c|c|c|c|c|c|c|}
\hline & \multirow[b]{2}{*}{ B } & \multirow[b]{2}{*}{ E.S } & \multirow[b]{2}{*}{ Wald } & \multirow[b]{2}{*}{ ddl } & \multirow[b]{2}{*}{ Sig. } & \multirow[b]{2}{*}{$\operatorname{Exp}(B)$} & \multicolumn{2}{|c|}{$\begin{array}{l}\text { Intervalle de } \\
\text { confiance } 95 \% \\
\text { pour } \operatorname{EXP}(\mathrm{B})\end{array}$} \\
\hline & & & & & & & & $\begin{array}{l}\text { Inférie } \\
\text { ur }\end{array}$ & $\begin{array}{c}\text { Supérieu } \\
\mathrm{r}\end{array}$ \\
\hline \multirow[t]{9}{*}{ Pas $1^{4}$} & $\begin{array}{l}\text { soutien_matériel_fina } \\
\mathrm{n}\end{array}$ & 1,414 & ,611 & 5,362 & 1 & 0,021 & 4,111 & 1,243 & 13,605 \\
\hline & Cons_avis & 4,830 & 1,207 & 16,021 & 1 & 0,000 & 125,259 & 11,764 & 1333,683 \\
\hline & $\begin{array}{l}\text { Communication_régul } \\
\text { ière }\end{array}$ & 1,906 & ,645 & 8,740 & 1 & 0,003 & 6,726 & 1,901 & 23,798 \\
\hline & $\begin{array}{l}\text { association_encadrée( } \\
\text { 1) }\end{array}$ & 3,321 & 1,229 & 7,304 & 1 & 0,007 & 27,695 & 2,491 & 307,910 \\
\hline & $\begin{array}{l}\text { Projection projet- } \\
\text { avenir }\end{array}$ & 2,144 & 1,205 & 6,548 & 1 & 0,007 & 8,533 & 2,478 & 85,158 \\
\hline & Sexe enquêté(1) & $-1,514$ & 1,296 & 1,364 & 1 & 0,243 & ,220 &, 017 & 2,792 \\
\hline & Âge enquêté &,- 038 &, 093 &, 172 & 1 & 0,679 &, 962 &, 802 & 1,154 \\
\hline & Niv étude enquêté & 2,005 & ,689 & 8,455 & 1 & 0,004 & 7,424 & 1,922 & 28,677 \\
\hline & Constante & $-22,103$ & 5,054 & 19,129 & 1 & 0,000 &, 000 & & \\
\hline
\end{tabular}

Source: Logiciel SPSS, Version 22.0

Dans le tableau ci-dessus, on note que les variables " sexe " et "âge " ne participent pas significativement à la survenue du comportement de résilience, car $\mathrm{P}>0,05$. Cependant le niveau d'études et l'élaboration de projet d'avenir participent significativement à la qualité du modèle.

\footnotetext{
${ }^{4}$ Introduction des variables au pas 1 : Sexe_enquêté, Âge_enquêté, Niv_étude_enquêté.
} 
On note par ailleurs que la chance d'être résilient pour le jeune qui se projette dans l'avenir est 6,548 plus important que celui qui n'a aucun projet d'avenir; pour celui qui a un niveau d'étude plus élevé la chance de ne pas passer à l'acte est 8,455 plus élevé que ceux qui un faible niveau d'études. En dehors des deux variables (sexe et âge), les variables explicatives ont simultanément une influence sur la probabilité de survenue de la résilience chez les jeunes. Autrement dit, le fait de développer des aptitudes de résilience n'est pas forcément tributaire ni du sexe ni de l'âge de l'individu. Ainsi, que l'on appartienne au sexe féminin ou au sexe masculin, il est susceptible de faire preuve de résilience. Autrement dit, on ne peut identifier de manière spécifique les jeunes résilient à la délinquance, en toute objectivité, à travers les variables "âge", et "sexe".

\subsubsection{Personnalité des jeunes résilients}

L'identification des différents traits de personnalité a été possible à l'aide d'un questionnaire de personnalité. En effet, sur l'ensemble des sujets (10) soumis audit questionnaires, seulement un seul, soit $10 \%$, présente une personnalité antisociale. Ce type de personnalité se distingue des autres, d'autant plus que les sujets de personnalité antisociale ont pour caractéristique essentielle le développement d'un comportement irresponsable et antisocial. Ils ne disposent d'aucune qualité principale ${ }^{5}$. Autrement dit, la plupart des jeunes résilients auquel le test de personnalité a été soumis, développe rarement un comportement antisocial. Cependant, la grande majorité des individus enquêtés $(90 \%)$ ne présentent aucun trait de personnalité. Les traits de personnalité les plus mis en exergue par les enquêtés sont les personnalités évitante $(80 \%)$, dépendante $(30 \%)$, obsessionnelle-compulsive $(20 \%)$, passive-agressive $(20 \%)$ et historique $(20 \%)$.

La personnalité évitante a pour caractéristique fondamentale, le mode général de timidité et de gêne dans les relations sociales. Le second trait de personnalité le plus observé chez les sujets qu'est la personnalité dépendante a comme caractéristique essentielle un comportement dépendant et soumis. Ce trait de personnalité est associé aux traits de personnalité évitante, historique et schizotypique ou narcissique. Quant aux personnalités obsessionnellecompulsive, passive-agressive qu'on retrouve également en général chez les sujets, elles ont pour caractéristiques principales le mode général de perfectionnisme, de rigidité et de résistance passive aux demandes de performances. Aussi est-il utile de faire mention de la réponse émotionnelle excessive et la recherche systématique d'attentions, d'éloges, caractéristique particulière de la personnalité histrionique ou hystérique.

\footnotetext{
${ }^{5}$ Association Américaine de Psychiatrie (APA), édition Masson.
} 
En dépit des caractéristiques asociales sus-évoquées, il est utile de souligner que la plupart des sujets développent quelques qualités principales, en l'occurrence, la discrétion, la réserve, la conscience professionnelle, les facilités de travailler d'une manière méthodique, accepter et exécuter les ordres, l'autonomie (la capacité à travailler seul) et l'adaptation à la routine, des facilités pour accepter, exécuter les ordres, déléguer des responsabilités, informer, aider les autres, recueillir des conseils et ont une aisance de contacts et des relations.

$\mathrm{Au}$ regard de cette analyse, l'on peut retenir que les ressources personnelles dont dispose la majorité des sujets peuvent être soit positives soit négatives. Il en de même pour les ressources sociales dont il bénéficie également. Par conséquent, c'est la combinaison de ces deux types de ressource, à savoir, personnelle et sociale voire relationnelle qui permet de définir la capacité de résilience chez un sujet donné. C'est le cas de P.E, 30 ans ingénieure informaticienne, l'une des personnes enquêtées qui affirme avoir perdu ses parents biologiques à l'âge de 8 ans. Son témoignage est le suivant :

Je suis fille unique de mes parents. Et du vivant de ceux-ci nous vivions dans un quartier résidentiel, étant donné que mes parents étaient tous deux fonctionnaires d'Etat. A la maison, je ne manquais de rien. J'avais tout, en tout cas presque tout à ma disposition (véhicule pour nous déposer à l'école, argent de poche, outil informatique à notre disposition, etc.). De tout ce confort, j'en ai bénéficié jusqu'à ce qu'un jour j'apprenne le décès de mes parents à la suite d'un grave accident de circulation. Depuis lors, je fus recueillie par l'une de mes tantes qui réside dans un quartier précaire à Abobo. Franchement, je vous avoue que ce fut un changement de vie radicale. Dans les débuts, cela n'a pas été du tout facile. J'avais l'impression que le ciel me tombait sur la tête. J'ai même voulu abandonner les études. A un moment donné, je me suis ressaisi et je me suis résolu à me battre pour réussir dans la vie. Malgré la précarité du quartier dans lequel je vivais, j'avais pris l'engagement de ne pas me livrer à la prostitution comme ce fut le cas de bon nombre de filles à l'époque. J'ai voulu coûte que coûte réussir pour faire honneur à la mémoire de mes défunts parents. Aujourd'hui, j'ai pu réaliser ce rêve et je suis Ingénieure informaticienne. Je remercie en toute sincérité ma tante, qui, bien qu'elle ait vécue dans un quartier précaire, faute de moyens, m'a toujours inculqué des valeurs pro sociales.

A la suite de ce témoignage, l'on peut retenir qu'il est possible de surmonter les difficultés auxquelles on peut être confrontées et réussir dans la vie, comme ce fut le cas de ce sujet. Cette enquêtée a recouru non seulement à des ressources personnelles mais également à celles que sa tante lui avait mises à disposition. 


\subsection{Caractéristiques des familles des jeunes résilients}

\subsubsection{Statut socioprofessionnel des parents}

Les résultats de cette étude révèlent que les jeunes qui ne commettent pas d'actes de délinquance dans la commune d'Abobo n'appartiennent pas à des familles ayant une situation matrimoniale particulière. A.M, 18ans, étudiante, précise : «ces enfants qui ne commettent pas d'actes criminels, sont issus de toutes les couches matrimoniales». Les parents de ces jeunes appartiennent à toutes les catégories, c'est-à-dire qu'ils peuvent aussi bien être mariés, divorcés, célibataires ou veuf (ves). Le cas de O.S, 16ans, élève en classe de première $\mathrm{D}$, interviewé au COM corrobore les propos de l'enquêté précédent. Son témoignage est le suivant :

Je suis issu d'une famille aisée. J'ai deux sœurs. Je suis en classe de $1^{\text {ère }}$ D. Mon père est banquier et ma mère est professeure de lycée dans le privé. En réalité, j'ai tout ce qu'il me faut à la maison, surtout en termes de soutien matériel et même affectif, d'autant plus que même dans ma chambre à coucher je dispose d'une télévision et d'un confort minimum. Je suis arrivé au COM suite à un vol commis à l'égard de ma mère, qui m'y a elle-même amené. En effet, $j$ 'ai soustrait frauduleusement, sur le compte bancaire de ma mère la somme de 675000 FCFA pour résoudre une contrainte financière que je lui avais exprimé. Aussi, à la maison ma mère me considère encore comme 'un bébé' et me fait subir beaucoup de privation (restriction des sorties, des heures de sortie, etc.). Ce que mes amis me reprochent tout le temps. Ainsi, afin d'intégrer la bande j'ai dû prendre des habitudes et me comporter comme les autres, c'est-à-dire, fumer de la drogue, courtiser les filles (rire), boire de la boisson forte... ». Un autre enquêté F.P., qui s'est adonné à la pratique du broutage, ajoute : «ce n'est pas parce qu'on vient d'une famille aisée qu'on ne commet pas d'actes délictueux. Nous-mêmes, nous en sommes des exemples réels. Ce qui nous a conduit à la délinquance, c'est l'effet d'imitation des amis, c'est-à-dire, l'envie de vouloir faire comme eux, de s'affirmer, d'être autonome.

Cependant, il est à noter que les jeunes qui ne commettent pas d'actes criminels appartiennent en général à des familles où les parents sont mariés et où il règne une relative harmonie familiale. C'est le cas par exemple de Y.S, l'un des jeunes pensionnaires du COM avec qui nous nous sommes entretenus, qui lui, n'a pas eu 'la chance' de vivre dans un cadre familial harmonieux.

Celui-ci, c'est-à-dire, Y.S, 18ans, élèves en classe de troisième affirme :

Je suis le seul enfant que mes parents ont conçu de leur union, même si de part et d'autre chacun des deux a eu des enfants avant leur

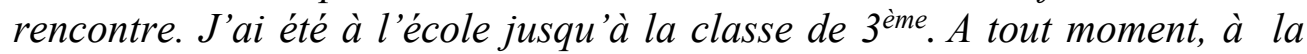
maison, mon père battait ma mère violemment. Impuissant face à cette situation, j'ai préféré quitter la cour familiale pour vivre dans la rue, où j'ai 
appris à fumer de la drogue, communément appelée dans notre cercle d'amis 'S4'6. C'est alors qu'un jour, lorsque nous nous y appliquions dans un fumoir, je fus arrêté et déféré par la suite au COM.

Cette situation traduit combien de fois l'harmonie familiale devrait être priorisée au sein du couple afin de permettre une bonne éducation des enfants. Le récit de ces enquêtés dénote qu'ils n’ont bénéficié d'aucune ressource familiale, nécessaire pour bâtir une bonne résilience.

\subsubsection{Niveau d'étude des parents}

L'étude apporte une précision sur le niveau d'étude des parents de jeunes résilients dans les quartiers défavorisés à Abobo. Les parents de ces jeunes ont un niveau d'étude relativement élevé. Ce niveau d'étude leur permet certainement d'être plus regardant sur le comportement de leurs enfants et de mieux les encadrer. affirme :

L'un des parents (infirmier d'Etat) rencontrés au cours de l'étude

Chez moi à la maison, je n'autorise à aucun de mes enfants de rentrer à des heures tardives. A partir de 22 heures, tout le monde doit être à la maison. Il y a des règles qui y sont établies pour le bon fonctionnement de la vie en famille. Je ne manque point de leur prodiguer des conseils à chaque fois que l'occasion se présente, surtout que moi-même j'ai dû batailler dure pour réussir dans la vie. Avec cette manière de procéder, mes enfants ont toujours eu des comportements exemplaires. De ce point de vue, la probabilité qu'un enfant rencontre des problèmes d'adaptation sociale au cours de son développement dépend en grande partie de la nature des interactions entre ses caractéristiques individuelles et les caractéristiques de l'environnement physique et social au sein duquel il grandit.

\section{Etude de relations entre des variables étudiées}

Cette étude de relations entre les variables nous permet de connaitre l'influence des différentes variables étudiées précédemment sur l'objet d'étude qu'est la résilience au passage à l'acte délictueux chez des jeunes. Il s'agit d'une manière plus précise de confronter ces variables aux différents indicateurs de la résilience.

\subsection{Capacité à demander de l'aide favorisée par l'interaction de plusieurs variables}

La capacité à demander de l'aide est fondamentale dans l'explication de la résilience au passage à l'acte délictueux chez certains jeunes. Et ce,

\footnotetext{
${ }^{6}$ Selon les propos de l'enquêté, ce code symbolise le cannabis, qui selon lui, est beaucoup prisé par les jeunes à Abobo, comme dans la plupart des communes de la ville d'Abidjan.
} 
d'autant plus que cette aptitude à demander de l'aide fait en général défaut aux délinquants. Les tableaux ci-dessous mettent en évidence l'interaction de plusieurs variables, favorisant cette aptitude à demander de l'aide chez certains jeunes vivant dans les quartiers défavorisés à Abobo.

$\underline{\text { Tableau 2 }}$ : Liaison entre 'Personne à qui l'on demande de l'aide' et Capacité à demander de l'aide'

\begin{tabular}{|c|c|c|c|c|}
\hline \multirow{2}{*}{\multicolumn{2}{|c|}{ Variable indépendante }} & \multicolumn{2}{|c|}{$\begin{array}{c}\text { Capacité à demander } \\
\text { de l'aide }\end{array}$} & \multirow[t]{2}{*}{ Total } \\
\hline & & Oui & Non & \\
\hline \multirow{4}{*}{$\begin{array}{l}\text { Personne à qui } \\
\text { on demande de } \\
\text { l'aide }\end{array}$} & $\begin{array}{l}\text { mes parents (père, } \\
\text { mère) }\end{array}$ & $44,8 \%$ & $3,6 \%$ & $48,5 \%$ \\
\hline & Amis & $8,8 \%$ & $0,5 \%$ & $9,3 \%$ \\
\hline & frères et sœurs & $7,7 \%$ & $0,5 \%$ & $8,2 \%$ \\
\hline & $\begin{array}{l}\text { toute autre } \\
\text { personne }\end{array}$ & $32,0 \%$ & $2,1 \%$ & $34,0 \%$ \\
\hline \multirow{2}{*}{\multicolumn{2}{|c|}{ Total }} & $93,3 \%$ & $6,7 \%$ & $100,0 \%$ \\
\hline & & Valeur & Ddl & $\begin{array}{l}\text { Signification asymptotique } \\
\text { (bilatérale) }\end{array}$ \\
\hline \multicolumn{2}{|c|}{ Khi-deux de Pearson } & 1,711 & 6 & ,944 \\
\hline
\end{tabular}

Source : Enquête, 2016

Les statistiques de ce tableau révèlent qu'une proportion non négligeable des jeunes non criminels (44,8\%) qui demandent de l'aide lorsqu'ils sont en difficulté, ont le plus souvent recours à leurs parents, c'està-dire, père et mère. De même, une autre proportion considérable $(32 \%)$ de ces jeunes, lorsqu'ils sont confrontés à des situations complexes demandent de l'aide à toute autre personne susceptible de leur venir en aide. Toutefois, seulement $16,5 \%$ de l'ensemble des personnes interrogées, lorsqu'ils sont en difficulté, demandent de l'aide soit à leurs amis $(8,8 \%)$ soit à leurs frères et sœurs (7,7\%). Cela témoigne de ce que la société et surtout des parents contribuent inéluctablement à développer chez ces jeunes cette capacité à demander de l'aide lorsqu'ils sont en difficulté. Et ce, d'autant plus que ceuxci en général, sont faciles d'accès ou d'approche. A cet effet, l'un des parents enquêté affirme : "Je suis beaucoup ouvert avec mes enfants, et on discute de tout sans tabou. Ce qui fait que, lorsqu'ils ont le moindre souci, ils m'en parlent ou en discutent avec leur mère (...) ». Ceci dit, si les parents ou la société sont hostiles à l'égard des enfants ou des jeunes, il leur serait difficile de solliciter leurs aides.

Par ailleurs, les résultats indiquent qu'il n'y a pas de lien entre la capacité à demander de l'aide et le type de personne à qui la demande est faite. 
Ce manque de lien s'explique par les éléments suivant : la probabilité $\mathrm{p}$ (significations bilatérales) $=0,944$ est supérieur à 0,05 et le Khi-deux de Pearson calculé $(1,711)$ est inférieur au Khi-deux seuil $(12,59)$ au seuil de confiance de $95 \%$. L'on peut en déduire que le type de personne à qui l'on demande de l'aide n'influence pas forcément la capacité à le faire.

En outre, la capacité à demander de l'aide chez les jeunes qui ne commettent pas d'actes criminels dans les quartiers précaires à Abobo, est également mise en lien avec le soutien matériel et financier, les conseils et avis dont ils bénéficient et la communication régulière et constante avec leurs parents.

Tableau 3: Liaison entre 'avis et conseils dont bénéficient certains jeunes' et 'capacité à demander de l'aide'

\begin{tabular}{|c|c|c|c|c|}
\hline \multirow{2}{*}{\multicolumn{2}{|c|}{ Variable indépendante }} & \multicolumn{2}{|c|}{$\begin{array}{c}\text { Capacité à demander de } \\
\text { l'aide }\end{array}$} & \multirow[t]{2}{*}{ Total } \\
\hline & & Oui & Non & \\
\hline \multirow{2}{*}{$\begin{array}{l}\text { Bénéficier de } \\
\text { conseils et d'avis }\end{array}$} & Jamais ou rarement & $9,0 \%$ & $1,0 \%$ & $10,0 \%$ \\
\hline & Souvent ou toujours & $80,6 \%$ & $9,4 \%$ & $90,0 \%$ \\
\hline \multicolumn{2}{|r|}{ Total } & $89,6 \%$ & $10,4 \%$ & $100,0 \%$ \\
\hline & & Valeur & Ddl & $\begin{array}{l}\text { Signification asymptotique } \\
\text { (bilatérale) }\end{array}$ \\
\hline \multicolumn{2}{|c|}{ Khi-deux de Pearson } & $1,488^{a}$ & 3 &, 685 \\
\hline
\end{tabular}

Source : Enquête, 2016

Selon les données de ce tableau, la majorité $(80,6 \%)$ de l'ensemble des jeunes non criminels interrogés, demandent de l'aide lorsqu'ils sont en difficulté, mais également bénéficient souvent ou toujours de conseils, d'avis ou d'informations sur la façon de faire quelque chose. Seulement $9 \%$ des personnes interrogées déclarent demander de l'aide lorsqu'ils sont en difficulté, sans toutefois bénéficier de conseils et d'avis sur la manière d'entreprendre quelque chose. Par ailleurs, une minorité (10,4\%) d'entre les personnes interrogées affirme ne pas demander de l'aide lorsqu'elles sont en difficulté. Parmi celle-ci, seulement 9,4\% bénéficient souvent ou toujours de conseils, d'avis ou d'informations sur la façon de faire quelque chose, tandis qu'une proportion relativement faible (1\%) affirme ne jamais en bénéficier ou rarement.

La signification $\mathrm{p}$ (significations bilatérales) $=0,685$ est supérieure à 0,05 , donc le test n'est pas significatif. L'hypothèse nulle H0 est conservée, c'est-à-dire qu'il n'y a pas forcément de lien entre les conseils et les avis dont on bénéficie et la capacité à demander de l'aide.

Si les conseils, les avis ou les informations, dont bénéficient certains jeunes sur la façon de faire quelque chose occupent une place de choix dans le développement de la capacité à demander de l'aide, il n'en est pas moins pour 
la communication régulière et constante avec les parents. Le tableau cidessous en est une illustration.

\subsection{Relation entre 'Réconfort et amour dont bénéficient certains jeunes' et 'Maîtrise de soi'}

Outre la capacité à demander de l'aide et la communication régulière et constante entre parents et enfants, la résilience au passage à l'acte délictueux requiert que l'individu qui en fait la preuve développe une bonne maîtrise de soi, d'autant plus que la maîtrise de soi est l'une des caractéristiques que l'on retrouve chez les non criminels.

Tableau 4 : Liaison entre réconfort et amour dont certains jeunes bénéficient' et 'maîtrise

\begin{tabular}{|c|c|c|c|c|}
\hline \multirow{2}{*}{\multicolumn{2}{|c|}{$\begin{array}{l}\text { Variable dépendante } \\
\text { Variable indépendante }\end{array}$}} & \multicolumn{2}{|c|}{ Maîtrise de soi } & \multirow[t]{2}{*}{ Total } \\
\hline & & Oui & Non & \\
\hline \multirow{2}{*}{$\begin{array}{l}\text { Bénéficier de } \\
\text { réconfort, d'amour }\end{array}$} & Jamais ou rarement & $12,6 \%$ & $3,5 \%$ & $16,1 \%$ \\
\hline & Souvent ou toujours & $59,3 \%$ & $24,6 \%$ & $83,9 \%$ \\
\hline \multirow{2}{*}{\multicolumn{2}{|c|}{ Total }} & $71,9 \%$ & $28,1 \%$ & $100,0 \%$ \\
\hline & & Valeur & Ddl & $\begin{array}{l}\text { Signification asymptotique } \\
\text { (bilatérale) }\end{array}$ \\
\hline \multicolumn{2}{|l|}{ Khi-deux de Pearson } &, $740^{\mathrm{a}}$ & 1 &, 390 \\
\hline
\end{tabular}

Source : Enquête, 2016

Ce tableau montre que la majorité des jeunes interrogés $(59,3 \%)$, non seulement parviennent à se contenir lorsqu'ils sont réellement en colère, mais aussi et surtout bénéficient souvent ou toujours de réconfort et d'amour et de protection lorsqu'ils traversent des moments difficiles, de maladie ou lorsque leur survient un coup dur. Cependant, une proportion relativement faible $(12,6 \%)$ des jeunes interrogés affirment se maîtriser lorsqu'ils sont en colère. Et ce bien qu'ils ne bénéficient jamais ou rarement de réconfort ou d'amour.

Par ailleurs, une proportion relativement considérable $(28,1 \%)$ de l'ensemble des jeunes interrogés affirme ne pas se contrôler lorsqu'ils sont en colère. Parmi ces jeunes, seulement 3,5\% estiment ne jamais bénéficier de réconfort ou d'amour et une autre proportion non négligeable (24,6\%) déclare être pourvu de réconfort et d'amour, lorsqu'ils traversent des moment difficiles. Le tableau ci-dessous précise que la signification $\mathrm{p}$ (significations bilatérales $)=0,390$ étant supérieure à 0,05 et le Khi-deux calculé $(0,740)$ étant inférieur au Khi-deux seuil $(3,84)$, le test n'est pas significatif. L'hypothèse nulle $\mathrm{H} 0$ est conservée, c'est-à-dire qu'il n'y a pas forcément de lien entre le réconfort, l'amour dont bénéficie certains jeunes vivant dans les quartiers défavorisés de la commune d'Abobo et leur maîtrise de soi.

Dans cette même perspective, le tableau ci-dessous met en évidence la relation entre l'attitude des jeunes lorsqu'ils sont en colère et leur maîtrise de soi. 
$\underline{\text { Tableau } 5}$ : Liaison entre 'Attitude des jeunes lorsqu'ils sont en colère contre une personne' et 'Maîtrise de soi'

\begin{tabular}{|c|c|c|c|c|}
\hline \multirow{2}{*}{\multicolumn{2}{|c|}{\begin{tabular}{l|l} 
dépendante & Variable \\
Variable indépendante & \\
\end{tabular}}} & \multicolumn{2}{|c|}{ Maîtrise de soi } & \multirow[t]{2}{*}{ Total } \\
\hline & & Oui & Non & \\
\hline \multirow{3}{*}{$\begin{array}{l}\text { Attitude des } \\
\text { jeunes lorsqu'ils } \\
\text { sont en colère } \\
\text { contre une } \\
\text { personne }\end{array}$} & $\begin{array}{l}\text { J'essaie de contenir } \\
\text { ma colère }\end{array}$ & $40,9 \%$ & $10,8 \%$ & $51,7 \%$ \\
\hline & $\begin{array}{l}\text { Je réagis pour } \\
\text { exprimer ma colère }\end{array}$ & $8,4 \%$ & $10,8 \%$ & $19,2 \%$ \\
\hline & $\begin{array}{l}\text { J'évite toute } \\
\text { discussion avec cette } \\
\text { personne }\end{array}$ & $21,2 \%$ & $7,9 \%$ & $29,1 \%$ \\
\hline \multirow{2}{*}{\multicolumn{2}{|c|}{ Total }} & $70,4 \%$ & $29,6 \%$ & $100,0 \%$ \\
\hline & & Valeur & Ddl & $\begin{array}{l}\text { Signification asymptotique } \\
\text { (bilatérale) }\end{array}$ \\
\hline \multicolumn{2}{|c|}{ Khi-deux de Pearson } & $17,409^{\mathrm{a}}$ & 2 &, 000 \\
\hline
\end{tabular}

Source : Enquête, 2016

$\mathrm{Au}$ regard des statistiques présentées par ce tableau, une proportion considérable $(40,9 \%)$ de l'ensemble des jeunes interrogés, qui estime développer une maîtrise de soi, font l'effort de se contenir lorsqu'ils sont en colère contre une personne. De même, une autre proportion non négligeable $(29,6 \%)$ des personnes interrogées, ayant une maîtrise de soi, évite toute discussion avec une personne contre laquelle elles sont en colère $(21,2 \%)$ et réagit pour exprimer son mécontentement $(8,4 \%)$. En outre, sur l'ensemble des jeunes interrogés, la majorité $(51,7 \%)$ affirme contenir sa colère et une autre grande majorité $(70,4 \%)$ des personnes interrogées déclare avoir une maîtrise de soi.

La probabilité $\mathrm{p}$ (signification bilatérale) $=0$ est inférieure à 0,05 et le Khi-deux de Pearson calculé $(17,04)$ est supérieur au Khi-deux seuil $(5,99)$ au seuil de confiance de $95 \%$. Par conséquent, l'on peut rejeter l'hypothèse nulle $\mathrm{H} 0$ et conserver l'hypothèse alternative $\mathrm{H} 1$, c'est-à-dire, qu'il y'a un lien entre l'attitude des jeunes lorsqu'ils sont en colère contre une personne et leur maîtrise de soi.

En d'autres termes, la plupart des jeunes qui résistent au passage à l'acte dans la commune d'Abobo, font l'effort de se contenir lorsqu'ils sont en colère contre une personne. Cette maîtrise de soi s'explique aussi en partie par le degré de participation du jeune à des activités religieuses.

\section{IV/ DISCUSSION ET CONCLUSION}

Les résultats de cette étude renferment quelques faiblesses, dues aux instruments de collecte de données, à l'échantillon d'enquête, aux conditions 
dans lesquelles se sont déroulées ces collectes d'informations, au traitement des données collectées et à l'interprétation des résultats obtenus. Il faut se garder de les généraliser de façon hasardeuse. Cependant il ne faut pas les rejeter du revers de la main.

Ces résultats mettent en exergue les caractéristiques psychosociales des jeunes résilients de la commune d'Abobo, tout en indiquant que les jeunes qui font preuve de résilience à la délinquance dans la commune sont de tous les âges et des deux sexes. L'on ne peut les identifier de manière spécifique, en toute objectivité, à travers les variables "âge', et "sexe'. La résilience peut donc se constater à tout âge. Les résultats de cette étude corroborent ceux de Anaut (Op.cit.), qui affirme que la résilience n'est pas acquise une fois pour toutes, mais en constant développement et l'on observe l'émergence du processus résilient à tout âge, y compris dans la vieillesse. Des recherches menées en Suède et en Belgique mettent en évidence que le niveau de la résilience augmente avec l'âge. En outre, l'étude soutient que la résilience au passage à l'acte délictueux chez certains jeunes vivant dans les quartiers précaires de la commune d'Abobo s'explique par des habiletés sociales acquises au sein de leurs familles (Sameroff, Op.cit.). Il s'agit de la capacité à demander de l'aide, la maîtrise de soi, du développement d'un sentiment d'autonomie et d'une projection dans l'avenir. Ces résultats confirment ceux des travaux de Masten (Op.cit.), Gonnet, Koffi et Cyrulnik (2010). Ces auteurs soutiennent que certains enfants font preuve de résilience en fonction de ressources auxquelles ils ont accès au sein de leur famille. Aussi précisent-ils que l'attachement et la confiance en soi développée chez l'enfant, sont dus à la mise à disposition de l'enfant d'une base de sécurité (Gonnet, Koffi \& Cyrulnik, Op.cit.). La résilience dépend donc des habiletés familiales. Cependant, ces habiletés sociales acquises au sein de la famille ne rendent pas totalement compte de la résilience au passage à l'acte délictueux chez des jeunes. Une autre étude serait nécessaire pour mettre en relief l'apport des soutiens sociaux dans le non passage à l'acte délictueux chez certains jeunes.

\section{References:}

1. Anaut, M. (2005) a. La résilience, surmonter les traumatismes. Lyon : Nathan université.

2. Anaut, M. (2005) b. Le concept de résilience et ses applications cliniques. Recherche en soins infirmiers. Vol.3 ( $\left.\mathrm{N}^{\circ} 82\right)$, p. 4-11. DOI 10.3917/rsi.082.0004.

3. Cyrulnik, B. (2003). Le murmure des fantômes. Paris : Odile Jacob.

4. Glaeser, E., Sacersote, B., \& Scheinkman, J. (1996). Crime and social interactions. Quarterly Journal of Economics, volume 111, $\mathrm{n}^{\circ} 2$.

5. Gonnet, G., Koffi, J.M. \& Cyrulnik, B. (2010). Résiliences, Cicatrices, Rébellion. Paris: L’Harmattan. 
6. Gralopois, M., Daluzeau, J. \& Oger, C. (2012). L'émergence $d u$ concept de résilience urbaine modifie-t-elle les pratiques de la prévention des risques en France? Polytech tours, Département aménagement.

7. Ionescu, S. (2012). Savoir prendre les tournants de la vie. http://santé.le figaro.fr/actualité.

8. Kouakou Oi Kouakou, B. (2016). La réussite paradoxale à l'école. Quand réussissent des élèves africains qui avaient peu de chance de réussir. Paris : L'Harmattan.

9. Luthar, S. (2013). La résilience chez les jeunes enfants et son importance sur leur développement psychosocial. New York: Encyclopédie sur le développement des jeunes enfants.

10. Maret, I. \& Cadoul, T. (2008). Résilience et reconstruction durable : que nous apprend La Nouvelle-Orléans? Annales de géographie, vol. 5, no 663, p. 104-124. 113

11. Masten, A. S. (2001). Ordinary magic: Resilience processes in development. American Psychologist, 56(3), 227-238. Http://Dx.Doi.Org/10.1037/0003-066X.56.3.227

12. N'goran, N. (2013). Circulation illicite des armes légères et criminalité violente dans la commune d'Abobo. Mémoire de Master1 en Criminologie (non publié) Abidjan : UFR Criminologie.

13. Sommer, K., Guiébo, J., Vignol, R., Maréchal, N., Sublet, M. \& Kuria, F. (2012). Côte d'Ivoire : profil urbain d'Abobo. Naïrobi : Publié par le Programme des Nations unies pour les établissements humains.

14. Terrise, B. \& Larose, F. (2001). La résilience: facteurs de risque et facteurs de protection dans l'environnement social et scolaire du jeune enfant. Publié dans Cahiers du Centre de recherche sur les formes d'éducation et d'enseignement, numéro thématique, École/Famille : Quelle médiation? XIV, P. 129-172.

15. Thomas, H. (2008). Vulnérabilité, fragilité, précarité, résilience, etc. De l'usage et de la traduction de notions éponges en sciences de l’homme et de la vie. Recueil Alexandries : coll. «Esquisses ». $\mathrm{n}^{\circ} 13$.

16. Tychey, C. (2001). Surmonter l'adversité : les fondements dynamiques de la résilience. Cahiers de psychologie clinique, vol. 16, no 1, p. 4968. 